
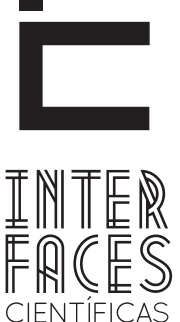

EXATAS E TECNOLÓGICAS

ISSN IMPRESSO - 2359-4934

E- ISSN - 2359-4942

DOI - 10.17564/2359-4934.2018v2n3p45-54

\title{
GENEALOGIA AMBIENTAL E PRODUÇÃO DO CONHECIMENTO INTERDISCIPLINAR
}

\author{
ENVIRONMENTAL GENEALOGY AND PRODUCTION OF INTERDISCIPLINARY KNOWLEDGE \\ GENEALOGÍA AMBIENTAL Y PRODUCCIÓN DEL CONOCIMIENTO INTERDISCIPLINAR
}

\author{
Carla Taciane Figueirêdo ${ }^{1}$ \\ Isabel Cristina Barreto Andrade ${ }^{3}$ \\ Arleide Barreto Silva
}

\author{
Antônio Vital Menezes de Souza ${ }^{2}$ \\ Roberto Rodrigues de Souza ${ }^{4}$
}

\section{RESUMO}

A produção do conhecimento nas ciências ambientais, nos últimos vinte anos, tem se caracterizado por uma polissemia teórica e metodológica. Assim, o campo das ciências ambientais pressupõe pesquisas rigorosas e politicamente engajadas numa dialogia na qual sejam explícitas a relações entre processo- pesquisador-objeto e problemáticas situadas em espaços de produção do conhecimento, dentre os quais se situam as ciências da natureza, as ciências humanas e sociais, as tecnologias. Faz-se necessário nortear esforços em direção do reconhecimento da compreensão de lacunas conceituais teóricas e metodológicas peculiares ao fazer cientifico. Nesse sentido esta pesquisa objetiva os estudos das raízes epistemológicas na produção de conhecimento nas ciências ambientais circunscritos na origem sociohistórica.
De natureza teórica com caráter epistemológico que se insere na área do conhecimento das ciências ambientais. Os autores que fundamentam esse estudo no tema das ciências ambientais Phillip Jr. (2000), referindo-se a genealogia Foucault (1984,1999, 2001, 2006, 2008), quanto à epistemologia Grayling (1996) e Japiassu (1992), na perspectiva do método Gil (1999) e Macedo (2006, 2007). Os procedimentos metodológicos utilizados norteiam-se pelo paradigma multirreferencial, os instrumentos de coleta de dados são a pesquisa bibliográfica e documental e análise será realizada através da análise do conteúdo. Os resultados obtidos contribuirão com a reflexão epistemológica na produção do conhecimento em ciências ambientais. A produção teórico-conceitual de fundamentos serve de subsidio futuras pesquisas em ciências ambien- 
tais em torno da categoria "genealogia ambiental"; a contribuição do estudo com uma abordagem interdisciplinar responde alguns questionamentos pertinentes na produção científica das ciências ambientais.

\section{ABSTRACT}

The production of knowledge in environmental science in the last twenty years has been characterized by a theoretical and methodological polysemy. Thus, the field of environmental science requires rigorous research and politically engaged in a dialogy in which either express the relationship between process-researcher and object problematic situated in knowledge production spaces, among which are situated the natural sciences, the humanities and social technologies. It is necessary to guide efforts towards the recognition of the understanding of theoretical and methodological conceptual peculiar gaps to make scientific. In this sense, this research aims to study the epistemological roots in the production of knowledge in environmental sciences at circumscribed socio-historical origin. Of theoretical with epistemological character that falls in the area of knowledge of environmental sciences. The authors underlying this study on the theme of environmental sciences Phillip Jr. (2000), referring to the genealogy Foucault (1984.1999, 2001, 2006, 2008), about the epistemology Grayling

\section{PALAVRAS-CHAVE}

Genealogia. Produção de conhecimento. Interdisciplinaridade. Ciências Ambientais.

(1996) and Japiassu (1992), in perspective of Gil method (1999) and Macedo $(2006,2007)$. The used methodological procedures are guided by multi-referential paradigm, data collection instruments are the bibliographical and documentary research and analysis will be performed by analyzing the content. The results will contribute to the epistemological reflection on the production of knowledge in environmental sciences. The theoretical and conceptual production foundations serves subsidy future research in environmental science around the category "Environmental genealogy"; the study's contribution to an interdisciplinary approach answers some pertinent questions in the scientific production of environmental sciences.

\section{KEYWORDS}

Genealogy. Production of knowledge. Interdisciplinarity. Environmental science

\section{RESUMEN}

La producción de conocimiento en ciencias ambientales en los últimos veinte años se ha caracterizado por una polisemia teórica y metodológica. Por lo tanto, el campo de las ciencias ambientales requiere una investigación rigurosa y políticamente comprometido en una dialogía en el que cualquiera exprese s relaciones entre el proceso-investigador-objeto y las problemáticas situadas en espacios de producción del conocimiento, entre los cuales se encuentran las ciencias naturales, las humanidades y tecnologías sociales. Es necesario orientar los esfuerzos hacia el reconocimiento de la comprensión de las brechas conceptuales 
teóricas y metodológicas peculiares del hacer científico. En este sentido, esta investigación tiene como objetivo estudiar las raíces epistemológicas en la producción de conocimiento en ciencias ambientales en origen socio-histórico circunscrito. De naturaleza teórica con carácter epistemológico que ingresa en el área de conocimiento de las ciencias ambientales. Los autores que subyacen a este estudio sobre el tema de las ciencias ambientales Phillip Jr. (2000), refiriéndose a la genealogía de Foucault (1984,1999, 2001, 2006, 2008), acerca de la epistemología Grayling (1996) y Japiassu (1992), en perspectiva del método Gil (1999) y Macedo (2006, 2007). Los procedimientos metodológicos utilizados son guiados por el paradigma multirreferencial, los instrumentos de recolección de datos son la investigación y el análisis bibliográfico y documental se lleva- rá a cabo mediante el análisis del contenido. Los resultados contribuirán a la reflexión epistemológica sobre la producción de conocimiento en ciencias ambientales. Las bases de producción teóricos y conceptuales sirve futuras investigaciones subsidio en la ciencia del medio ambiente alrededor de la categoría de "genealogía del Medio Ambiente”; la contribución del estudio para un enfoque interdisciplinario responde algunas preguntas pertinentes en la producción científica de las ciencias ambientales.

\section{PALABRAS CLAVE}

Genealogía. Producción de conocimiento. Interdisciplinariedad. Ciencias ambientales.

\section{INTRODUÇ̃̃̃O}

A produção do conhecimento nas ciências ambientais, nos últimos vinte anos, tem se caracterizado por uma polissemia teórica e metodológica. Assim sendo, o campo das ciências ambientais pressupõe pesquisas rigorosas e politicamente engajadas numa dialogia na qual sejam explícitas a relações entre processo- pesquisador-objeto e problemáticas situadas em espaços multirreferenciais de produção do conhecimento.

Por isso faz-se necessário voltar esforços na direção do reconhecimento da compreensão de lacunas conceituais teóricas e metodológicas peculiares ao fazer científico. As micropolíticas engendradas na produção do conhecimento interdisciplinar e as relações de saber-poder, implícitas na epistemologia das ciências ambientais, compreende o objeto desta pesquisa intitulada: Genealogia Ambiental e Produção do Conhecimento Interdisciplinar.

Neste contexto, as ciências ambientais contextualizam-se por um avanço desproporcional do conhecimento técnico e científico em relação à capacidade ético-moral quanto ao seu uso. Haja vista a discussão realizada por especialistas disciplinares, particularizada nas diversas áreas do conhecimento, que se expressam incoerentes e desconexas da realidade socioambiental contemporânea. Tais perspectivas incorrem em práticas seculares de apartheid existentes entre ciências naturais, exatas e humanas. Todavia, essa situação requer abordagens dialógicas e/ou complementares nas quais a premissa direciona-se à interconexão de distintos saberes e informações.

Por isso, uma perspectiva de construção de conhecimento metamórfico e descontínuo pode ser representado por uma figura rizomática, que ultrapasse 0 modelo interdisciplinar, direcionando-se a um paradigma transdisciplinar, por meio do qual a construção de conceitos se formalize mediante o intercambio e intersecção dos saberes oriundos de diferentes áreas e paradigmas. Nesta pesquisa, a história da ciência, sua contribuição e seu legado conjectural, volta-se à análise das micropolíticas engendradas na produção do conhecimento interdisciplinar nas ciências am- 
bientais quês e institucionalizam ao longo dos últimos vinte anos no cenário brasileiro.

A problemática de estudo desta pesquisa se explicita a partir de um vazio epistemológico, quanto à construção de conhecimentos teórico-metodológicos. Por isto a construção do conhecimento que ainda se ancora na classificação taxonômica dos objetos, das áreas de conhecimento, numa disciplinarização científica fragmentária e disjuntiva, necessita ser contextualizada como conjunto de práticas a serem superadas no cenário das ciências ambientais. Faz-se necessário, trazer à tona reflexões pontuais em torno das questões metodológicas na produção de conhecimento interdisciplinar em ciências ambientais.

Nesta pesquisa, tal problematização é de natureza epistemológica. A produção foucaultiana (1984; 1999; 2008) é referência fundamental para este trabalho, principalmente as inspirações do método genealógico. Assim, partindo do pressuposto conceito de genealogia se expressa como método, essa pesquisa subsidia-see na teoria foucaultiana, de modo que a problematização epistemológica referente ao saber das ciências ambientais decorre de uma construção cientifica norteada pelo questionamento ao conhecimento posto que se complementa numa tentativa de atualizar os saberes nomeados ambientais e transversalizá-los.

0 conhecimento produzido em ciências ambientais é amplamente legitimado por poderes microfísicos, invisibilizados na arena científica. Sendo assim, a origem dos saberes ambientais, as circunstâncias em que estão inscritos, são passíveis de estudo e análise. 0 objeto de estudo desta pesquisa refere-se as micropolíticas engendradas na produção do conhecimento interdisciplinar em ciências ambientais considerando a produção de conhecimentos por meio de publicações de teses, dissertações de autores variados dentro da demarcação temporal dos últimos vinte anos.

O desenvolvimento de uma pesquisa com o intuito de analisar a articulação dos mecanismos de poder e suas imbricações com as formas de saber científico, particularmente nas ciências ambientais, torna-se imprescindível. O objetivo geral foi analisar a institucionalização da produção do conhecimento interdisciplinar, tomando como ponto de partida as micropolíticas engendradas no campo das ciências ambientais.

Especificamente a caracterização da institucionalização da produção científica das ciências ambientais na área interdisciplinar, por meio da identificação e caracterização metodológicas empregadas e/ ou utilizadas nas pesquisas já desenvolvidas, analisando sua relevância, pertinência e significância epistemológica para o desenvolvimento da área.

Por conseguinte, é fundamental, após análise rigorosa das questões metodológicas formularem elementos de natureza teórico-conceitual que permitam trazer à tona a genealogia ambiental, como ferramenta teórico-metodológica que dinamize a produção do conhecimento interdisciplinar nas ciências ambientais.

Diante do exposto, o objeto de estudo desta pesquisa direciona-se tanto à análise da institucionalização da produção cientifica na esfera interdisciplinar em ciências ambientais, quanto se destina a elaboração conceitual do que se denomina genealogia ambiental. As categorias que fundamentam o estudo são genealogia, produção do conhecimento, interdisciplinaridade e ciências ambientais.

A transversalidade que permeia os conceitos e as categorias explicitadas possibilita o diálogo entre algumas áreas do conhecimento, dentre elas, pode-se indicar as ciências humanas, ciências sociais aplicadas e, principalmente, a fundamentação filosófica na concretização de uma visão sistêmica na abordagem das categorias, na resposta aos questionamentos e no cumprimento dos objetivos propostos.

Referindo-se ao conceito de genealogia, Foucault afirma que genealogia é

[...] uma inscrição dos saberes na hierarquia de poderes própria à ciência, um empreendimento para libertar da sujeição dos saberes históricos, de modo a torná-los capazes de oposição e de luta contra a coerção de um discurso teórico, unitário, formal e científico [...] contra a hierarquização científica do conhecimento e seus efeitos intrínsecos de poder [...]. (FOUCAULT, 1984, p. 172). 
Assim, a fundamentação nessas categorias denota o fio condutor teórico desta pesquisa. Numa perspectiva interdisciplinar e de construção de um conceito integrador, articulando diferentes áreas de conhecimento. Dessa forma as categorias genealogia e interdisciplinaridade explicitada prospectam a produção do conhecimento da genealogia ambiental que confluem conhecimentos, saberes, informações e configuram a complexidade do campo das ciências ambientais.

Por fim, a categoria da interdisciplinaridade caracteriza-se pela polissemia conceitual que implica na criticidade em defini-la. Fazenda (2008) destaca que

[...] a interação existente entre duas ou mais disciplinas pode-nos encaminhar da simples comunicação das ideias até a integração mútua dos conceitos-chave da epistemologia, da terminologia, do procedimento, dos dados e da organização da pesquisa e do ensino, relacionando-os.

\section{MÉTODO}

\subsection{PROCEDIMENTOS HEURÍSTICOS DA PESQUISA}

Esta pesquisa é de natureza fundamental e teórica. Preocupa-se em estudar as formas de produção e sistematização do conhecimento por meio da perspectiva epistemológica multirreferencial, numa complementaridade ao referencial metodológico da etnopesquisa crítica e o método genealógico foucaultiano. Respalda-se, pois, na busca de inovações teóricas e metodológicas que se fundamentam em associar conceitos e utilizar uma metodologia própria. Isto significa afirmar que, na abordagem multirreferencial a prática da pesquisa propicia a intercomunicação entre áreas distintas do conhecimento, idealizando-se atingir os objetivos propostos e responder aos questionamentos apresentados.

Os principais elementos formais (instrumentos e técnicas) são as produções teóricas conceituais sobre o conhecimento interdisciplinar e as ciências ambientais delimitadas nos últimos vinte anos, complementados pelos documentos e relatórios de órgãos oficiais recentes oriundos da "Calnter", Capes, Caamb.
Nesse sentido os autores que fundamentam esse estudo são, sobretudo, Foucault (1984, 1999, 2001,2006, 2008) na abordagem sobre genealogia, Phillip Jr. (2000), Fazenda (2008) no campo da interdisciplinaridade e das ciências ambientais, Macedo (2006) referindo-se etnopesquisa e multirreferencialidade. Nesse sentido a área de estudo se caracteriza por um campo epistemológico de fundamentação teórica, identificando na tessitura do poder os "nós" inteligíveis e necessários para o entendimento e enfrentamento do poder - saber.

Os instrumentos de coleta de informações se estruturam na pesquisa documental, na pesquisa bibliográfica dos referentes teóricos e posterior análise do discurso nos relatórios, artigos e produção conceitual sobre as categorias estudadas. A abordagem genealógica é pertinente, pois enquanto uma ontologia do presente e operacionalidade construída no plano fundamental com traços e tramas de sistematização do conhecimento explícitas dos documentos expressa com destaque na formação historiográfica.

Nesse caminho de busca das origens ontológicas do discurso ambientalista e os atores discursivos que preponderam e ressoam na produção do conhecimento das ciências ambientais, as considerações de Paul Ricoeuer apontam uma crítica nas pesquisas que analisam discurso, segundo o autor:

\begin{abstract}
[...] ainda falta explicitar o enunciador da enunciação. Sua designação se dá pelo recurso a dictícos, esses instrumentos da linguagem que se limitam a mostrar singularidades, transcendendo a especificação genérica; os pronomes pessoais, os advérbios de tempo e de lugar, as formas verbais, as descrições definidas são os meios ordinários de designação das quais depende a autodesignação dos sujeitos falantes. (RICOEUR, 2006, p. 111).
\end{abstract}

Os princípios epistemológicos que norteiam essa pesquisa são de natureza multirreferencial, Macedo (2006, p. 20) informa que "[...] na perspectiva fenomenológica e hermenêutica, a investigação do fenômeno humano encontra-se aberta ao acontecimento implicado ser-no-mundo-com o que além de pressupor o que já está predeterminado", lida de forma, pri- 
vilegiada, com a dimensão de projeto que caracteriza o homem-sendo-no-mundo-com o que nunca é, mas sempre está-sendo ultrapassagem.

Ainda de acordo com Macedo (2006, p. 93) a “[...] inspiração multirreferencial não está na afirmação da limitação dos diversos campos do saber, da tomada de consciência da necessidade de rigor fecundante, da nossa ignorância enquanto inquietação" e complementa a epistemologia multirrerferencial edifica-se a partir da "aceitação da irredutível complexidade da emergência humana, isto é do seu caráter indexal, opaco, reflexivo, temporal, molar, ideográfico, insuficiente, contraditório e eminentemente relacional."

A informação que mais se aproxima ao método genealógico apropriado para efetivação da pesquisa é o aporte teórico prático da epistemologia multirreferencial que se abre a pluralidade das referências, à alteridade, ao multiculturalismo, às contradições, ao dinamismo semântico às práxis, às insuficiências e emergências, para não perder o homem e sua complexidade, anulados na deificação da norma científica, como argumenta Macedo (2006, p. 94).

0 método como princípio, considerando a preponderância das ciências do homem na pesquisa se fundamenta pela "interrogação do próprio conhecimento a partir do conhecedor, do conhecido e do conhecível" Macedo (2006, p. 94) acrescenta que a problemática principal da etnopesquisa crítica e multirreferencial condizente com o método genealógico é a argumentação de que o acolhimento e a preocupação com as “múltiplas realidades”, com a natural diversidade das construções humanas, "[...] a etnopesquisa rejeita o pensamento nomonético cultivado na compulsão explicativa”, apreende no multiculturalismo uma fonte de pensamento basilar para suas fundações epistemológicas e metodológicas.

A base multirreferencial da pesquisa possui a subjetividade, alteridade autonomia como características e ideias mestras do conceito acima explicitas e subsidiadas por Macedo $(2006,2007)$ associam as concepções de indexação, de biodegrabilidade do conhecimento, constituindo-se eminentemente descritiva e sociopolítica, com demarcações da crítica científica, até porque a etnopesquisa não permite traição às memórias da formação acadêmica que engessaram disciplinarmente os fundamentos teóricos metodológicas de uma construção linear e monorreferencial marxista, entretanto essa opção metodológica implica numa transformação da própria visão de construção científica, pautada num discurso articulador, da complementaridade fincado na memória daquele que o produz, pesquisa como processo em contínua construção.

Ressalta-se que a complementaridade metodológica foi inevitável, pois a pesquisa exploratória casase como o tipo de abordagem realizada por se tratar de uma pesquisa de natureza fundamental ou teórica, que busca a construção de conceitos e teorias, o diálogo e explicitação de algumas categorias, a fiabilidade dos conceitos e teorias já consolidados, nessas relações que as obras mantém entre se pretender explicitar, a congruência e divergência desses conceitos e ideias acessíveis durante a pesquisa documental

\section{RESULTADOS E DISCUSSÕES}

Os principais resultados dessa pesquisa se fundamentam em três argumentos:

a) na contribuição com a reflexão epistemológica na produção do conhecimento em ciências ambientais de modo a favorecer aproximações com a filosofia foucaultiana a respeito da institucionalização na pesquisa em suas micropolíticas na esfera interdisciplinar;

b) na formulação por meio de uma produção teórico-conceitual de fundamentos que subsidiem futuras pesquisas em ciências ambientais em torno da categoria "genealogia ambiental";

c) contribui com uma abordagem interdisciplinar que tem propósito de responder alguns questionamentos pertinentes na produção científica das ciências ambientais.

A pertinência social e científica dessa pesquisa está na possível contribuição com a análise das ciências ambientais e das micropolíticas que institucionalizam esse campo de saber e do conhecimento científico, baseada na criticidade fundamental na 
academia e na construção da ciência. Essas contribuições corroboram com as prerrogativas do Programa de Pós-graduação em Desenvolvimento e Meio Ambiente (PRODEMA) da Universidade Federal de Sergipe, espaço acadêmico propício para produção de uma ciência crítica e problematizadora.

A formulação do conceito de genealogia ambiental, considerando que a formação doutoral e os programas de pós-graduação configuram-se como lócus de produção do conhecimento, é cientificamente relevante, pois contribuirá epistemologicamente com a área interdisciplinar, sua relevância se expressa diante da possível realização de posteriores estudos na temática ambiental.

Neste contexto, tendo como referência a genealogia numa concepção de ontologia do presente, essa contribuição se comprova mediante a expressão de um panorama, explicitando os referentes discursivos que estudam a área interdisciplinar, as micropolíticas e relações de poder saber invisibilizadas na produção de conhecimento dessa área.

Espera-se como resultado a formulação do conceito de genealogia ambiental e o subsídio a futuras pesquisas, considerando que o conhecimento produzido permitirá uma grafia descritiva dos fenômenos contextuais da produção do conhecimento interdisciplinar.

Entretanto, mesmo diante da proposta interdisciplinar de produção de conhecimento preponderantes nas ciências ambientais, viabilizar na prática de pesquisa com esses pressupostos tem algumas implicações e necessita uma formação contemporânea contínua aos cientistas ambiental que se predispõem à nova abordagem, postulados por uma realidade complexa, dinâmica e situada histórico, político e socialmente. Academicamente o exercício da docência do cientista ambiental necessita uma reflexividade da ação.

Concordamos com Lenoir \& Hasni (2004) quando afirmam que se deve conceber a interdisciplinaridade de forma a integrar estas três visões. Se algo entra por definição na prática interdisciplinar é a condição de que se deve buscar a complementação entre os diversos conhecimentos disciplinares.

Essa nova forma de produzir conhecimento traz à tona a incorporação da agenda pública na agenda cientifica, estabelecidas a partir de novas práticas, com diferentes linguagens metodológicas e conceituais. De acordo com Maranhão (2010) esse novo modo de produzir conhecimento caracteriza-se pela,

[...] interdisciplinaridade, empirismo, heterogeneidade institucional (empresas, governo, academia) e responsabilidade institucional do conhecimento, diferenciando-se do modelo tradicional, disciplinar, conhecimento teórico-acadêmico, homogêneo e hierárquico.

\begin{abstract}
Além de reunir vários especialistas de diferentes áreas do conhecimento, com diversos saberes especializados, a prática interdisciplinar de produção de conhecimento possibilita uma evolução da aplicação de conceitos e métodos de diferentes áreas, gerando algo que não se encaixa perfeitamente em nenhuma delas. Ou seja, a interdisciplinaridade surge no contexto da aplicação e necessita de difusão e comunicação de resultados parciais ao longo de um processo dinâmico de pesquisa. (MARANHÃO, 2010, p. 563, grifo da autora).
\end{abstract}

A consistência criteriosa e o rigor científico, próprio à ciência, seus critérios de produção de conhecimento não são explícitos no campo das ciências ambientais. 0 desenvolvimento do conhecimento prático, os saberes cotidianos o senso comum, inclusive da linguagem raramente são considerados, salvo em pesquisas fundamentadas na etnografia, no paradigma fenomenológico interpretativo crítico, ou histórico, comparativo onde se percebe a flexibilização nos instrumentos e técnicas de análise.

\section{CONCLUSÃO}

Outra abordagem explícita nas ciências ambientais se baseia na valoração econômica, principalmente nas temáticas de pesquisa que respondem aos questionamentos da problemática ambiental contemporânea, ou na ética ambiental enquanto princípio básico da prática científica no campo das ciências ambientais.

O fator determinante da interdisciplinaridade não pode ser buscado exclusivamente em torno das “necessidades" dos objetos, perguntando pelos seus 
significados nos planos ontológico e epistemológico; também as "necessidades" profissionais e sociais dos sujeitos não podem ser o fator determinante exclusivo; assim como tampouco as dimensões humanas intersubjetivas podem ser vistas como fator determinante exclusivo da interdisciplinaridade.

Como já mencionado, as fronteiras disciplinares acabam por restringir o âmbito da prática cientifica das ciências ambientais. A interdisciplinaridade, como suporte teórico-metodológico, não desconstrói a produção e organização do conhecimento disciplinar. Surge como opção na interpretação da problemática ambiental e viés para as ciências ambientais, campo genuinamente complexo do conhecimento.

\section{REFERÊNCIAS}

\section{DIEGUES, Antônio Carlos. 0 mito moderno da}

natureza intocada. São Paulo: NUPAUB-USP, 1994.

DIEGUES, Antônio Carlos. Etnoconservação novos rumos para a conservação da natureza. São Paulo: HUCITEC NUPAUB-USP, 2000.

FAZENDA, Ivani. (Org.). 0 que é

Interdisciplinaridade? São Paulo: Cortez, 2008.

FOUCAULT, Michel. Microfísica do poder. Rio de Janeiro: Graal, 1984.

FOUCAULT, Michel. A história da sexualidade II - 0 uso dos prazeres. Rio de Janeiro: Graal. 1984.
FOUCAULT, Michel. Em defesa da sociedade: curso no Collégge de France (1975-1976). São Paulo: Martins Fontes,1999.

FOUCAULT, Michel. Vigiar e punir: nascimento da prisão. Tradução de Raquel Ramalhete. 35.ed. Petrópolis. Rio de Janeiro: Vozes, 2008.

LEFF, Enrique. Complexidade, Interdisciplinaridade e Saber Ambiental. In: PHILLIP, J.R. A.

Interdisciplinaridade em ciências ambientais. São Paulo: Siginus, 2000.

LENOIR, Y.; HASNI, A. La interdisciplinaridad: por un matrimonio abierto de la razón, de la mano y del corazón. Revista Ibero-Americana de Educación, n.35, 2004.

MACEDO, Roberto Sidnei. A etnopesquisa crítica e multirreferencial nas ciências humanas e na educação. 2.ed. Salvador-BA: Edufba, 2006.

MACEDO, Roberto Sidnei. Currículo: campo, conceito e pesquisa. Petrópolis, RJ: Vozes, 2007.

RICOEUR, Paul. Percurso do reconhecimento. São Paulo: Loyola, 2006.

SACHS, Ignacy. Rumo a ecossocioeconomia: teoria e prática do desenvolvimento [Org. Paulo Freira Vieira. São Paulo: Cortez, 2007.

TRINDADE, D. Interdisciplinaridade: um novo olhar sobre as ciências. FAZENDA, I. (Org.). 0 que é interdisciplinaridade? São Paulo: Cortez, 2008. 
1 Doutoranda em Desenvolvimento e Meio Ambiente da Universidade Federal de Sergipe - UFS; Núcleo de pós Graduação em Desenvolvimento e Meio Ambiente, Universidade Federal de Sergipe - UFS; Bacharel em História. E-mail: carlatacyane@hotmail.com

2 Professor Adjunto da Universidade Federal de Sergipe - UFS; Doutor em Educação, Departamento de Educação. Líder do Grupo de Pesquisa em Tecnologias Intelectuais, Mídias e Educação Contemporânea - SEMINALIS, da Universidade Federal de Sergipe - UFS. E-mail: a.vmsouza@yahoo.com.br

3 Doutoranda em Desenvolvimento e Meio Ambiente, Programa de Pós-Graduação em Desenvolvimento e Meio Ambiente - PRODEMA da Universidade Federal de Sergipe - UFS; Bacharel em Administração. E-mail: icbandrade@ yahoo.com.br

4 Professor Doutor do Departamento de Engenharia Química e do Programa de Pós-Graduação em Desenvolvimento e Meio Ambiente - P RODEMA da Universidade Federal de Sergipe - UFS. E-mail: souza@ufs.br

5 Professora Mestra em Administração pela Universidade Federal da Paraíba (2003); Especialista em Administração Universitária - OUI/IGLU (2002); Especialista em Jornalismo Político e Econômico - FITS (1995); Especialista em Administração de Empresas - FITS (1993); Especialista em Administração e Gerência de Unidades de Ensino - FITS (1992); Licenciada Plena em Pedaduação da Universidade Tiradentes - UNIT. E-mail: arleide_barreto@unit.br 
